\title{
Spiral Taping Improves Performance on Star Excursion Balance Test in Individuals with Unilateral Chronic Ankle Instability
}

\author{
Young-Sook Bae \\ Department of Physical Therapy, College of Health Science, Gachon University, Seongnam, Korea
}

Purpose: This study was to investigate the short-term effects of spiral taping (ST) on performance on the star excursion balance test (SEBT) in individuals with unilateral chronic ankle instability (CAI).

Methods: This study was single-group pre - post measures experimental design. The subjects with CAl were 39 (range, 20-31 years; male 16, females 23) were enrolled in the study. The discomfort had in unilateral ankle and Cumberland ankle instability score was 19.56 ( \pm 3.29). Spiral tape (a width of $3 \mathrm{~mm}$ ) was applied $3 \times 4$ cross shape on medial malleolus, lateral malleolus and dorsal of talocural joint of unstable ankle. SEBT was measured baseline and $30 \mathrm{~min}$ later in stable ankle and unstable ankle.

Results: SEBT showed significantly improved after applying the $\mathrm{ST}(\mathrm{p}<0.05, \mathrm{ES}=0.74)$ on unstable ankle. In comparison the difference of stable and unstable ankle, between the pretest and posttest were significant differences $(p<0.01, E S=1.88)$.

Conclusion: These results indicated that ST improves performance on the SEBT. Therefore, it suggests that ST may be a suitable intervention to dynamic balance in patients with CAl.

Keywords: Chronic ankle instability, Star excursion balance test, Spiral taping

\section{INTRODUCTION}

Chronic ankle instability (CAI) is a condition where there is repetitive ankle instability, recurrent sprains, and diminished self-reported function in daily life. Symptoms of pain and instability of the ankle during daily activities are commonly present in CAI. ${ }^{2} \mathrm{Ac}$ cordingly, symptoms of pain, instability, and "giving way" are observed in approximately 55\%-75\% of patients after an initial ankle injury, and these symptoms can persist for 6-18 months following the initial injury. ${ }^{3,4}$ It has been suggested that CAI is determined by the correlation between mechanical and functional ankle insufficiencies, and it is believed that this correlation is based on the progression of CAI. ${ }^{3}$ Functional insufficiencies include impaired proprioception, impaired neuromuscular control, and impaired postural control. ${ }^{5}$ Furthermore, there are postural stability deficits in both static and dynamic situations in CAI. ${ }^{6}$ Dynamic balance is defined as maintaining balance while transitioning from a static to

Received Nov 18, 2016 Revised Dec 13, 2016

Accepted Dec 30, 2016

Corresponding author Young-Sook Bae

E-mail baeys@gachon.ac.kr dynamic state. ${ }^{7}$ Therefore, it is important for postural stability as dynamic balance deficits to be emphasized during rehabilitation. SEBT is reliable test for measuring dynamic balance. ${ }^{8}$ The SEBT is clinical test known to detect functional dysfunction associated with lower extremity pathology. ${ }^{9}$ Particularly, CAI was measured balance deficit by SEBT. ${ }^{10}$ Hong et a $^{11}$ reported neuromuscular training program for CAI enhanced STBT and the dynamic balance.

In addition, ankle taping such as Mulligan's fibular repositioning taping $^{12}$ and ankle balance taping with kinesiology taping ${ }^{13}$ are known to positively affect postural stability in CAI. The application of taping techniques is a common intervention used in ankle injuries. Recovery of dynamic balance after an orthopedic injury such as CAI is considered a major clinical rehabilitation goal. ${ }^{14}$

Ankle injury prevention and attenuation of recurring ankle injuries are important for limiting CAI. The way of reducing damage in the ankle joint is to apply external prophylactic support via ankle taping. Previous studies have suggested that taping the ankle joint is

Copylight (C2016 The Korea Society of Physical Therapy

This is an Open Access article distribute under the terms of the Creative Commons Attribution Non-commercial License (Http:// creativecommons.org/license/by-nc/4.o.) which permits unrestricted non-commercial use, distribution, and reproduction in any medium, provided the original work is properly cited. 
the most effective technique for reducing recurring ankle damage in patients with a history of ankle joint injury. ${ }^{14}$ Several types of ankle tapes can be used, including elastic and non-elastic tapes. Among the non-elastic tapes, the athletic tape, Mulligan's tape, and spiral taping (ST) developed by Dr. Nobudaka Tanaka, are used. ${ }^{15}$

The ST, which is referred to as a balance taping, is attached in a spiral direction after measuring electromagnetic flows through muscles and skin according to the principle of directivity and response points. ${ }^{16} \mathrm{ST}$ is known to be effective for low back pain, ${ }^{17}$ and balance is increased when applied on the ankle. ${ }^{18}$ As such, ST is frequently used in the clinic, the effect of ST on ankle injury is unclear.

The purpose of present study identified changes performance on the star excursion balance test (SEBT), which is known to detect dynamic balance, following application of the spiral taping in individuals with unilateral CAI.

\section{METHODS}

\section{Subjects}

Participants were 21 to 30 years old and were recruited from university students. Participants (male 16, female 23) with CAI were recruited. The mean age of the subjects was $22.44( \pm 2.66)$ years, mean height of $168.38( \pm 7.75) \mathrm{cm}$, weight of $64.46( \pm 9.76) \mathrm{kg}$.

Inclusion criteria for study participation were discomfort for six month over in unilateral ankle, Cumberland ankle instability score below 23 , have no swelling on ankle, able to support weight with an unstable ankle, and provision of written informed consent. Exclusion criteria were history of any surgery and fractured bones in the ankle within the past six months, Cumberland ankle instability score over 24, balance impairment or neurological or vestibular impairment; decreased sensation in the lower limb, any contraindications to the measurement procedures. All subjects provided written informed consent, and signed an informed consent approved by the Institutional Review Board.

A priori sample size calculations were performed using data from pilot study of eight participants. The software G-power 3.1.9.2 determined that thirty nine subjects were required, with a significance level of 0.05 , power 0.08 , and effect size of 0.8 (calculated using the mean and standard deviation from the pilot study).

\section{Intervention}

ST was applied by single physical therapist with 5 years over of experience. The participants were blind to the type of tape used. The size of the ST is width of $3 \mathrm{~mm}$ was used (SPX-50H, Nichiban Inc., Japan), ST is adhesive non-elastic tape. In this study, ST was applied as follows: (1) The tape was attached under the medial malleolus and then above and below it. The tape's direction was diagonal toward the right at a $30^{\circ}-35^{\circ}$ angle with an 8 - $\mathrm{mm}$ interval and attached the three tapes. Next, the top of the next tape attached was divided and its direction was diagonal toward the left at a $30^{\circ}-35^{\circ}$ angle with an 8 -mm interval and attached four tapes. As $3 \times 4$ shape, the tape was attached on the medial malleolus (Figure 1A). (2) Then,
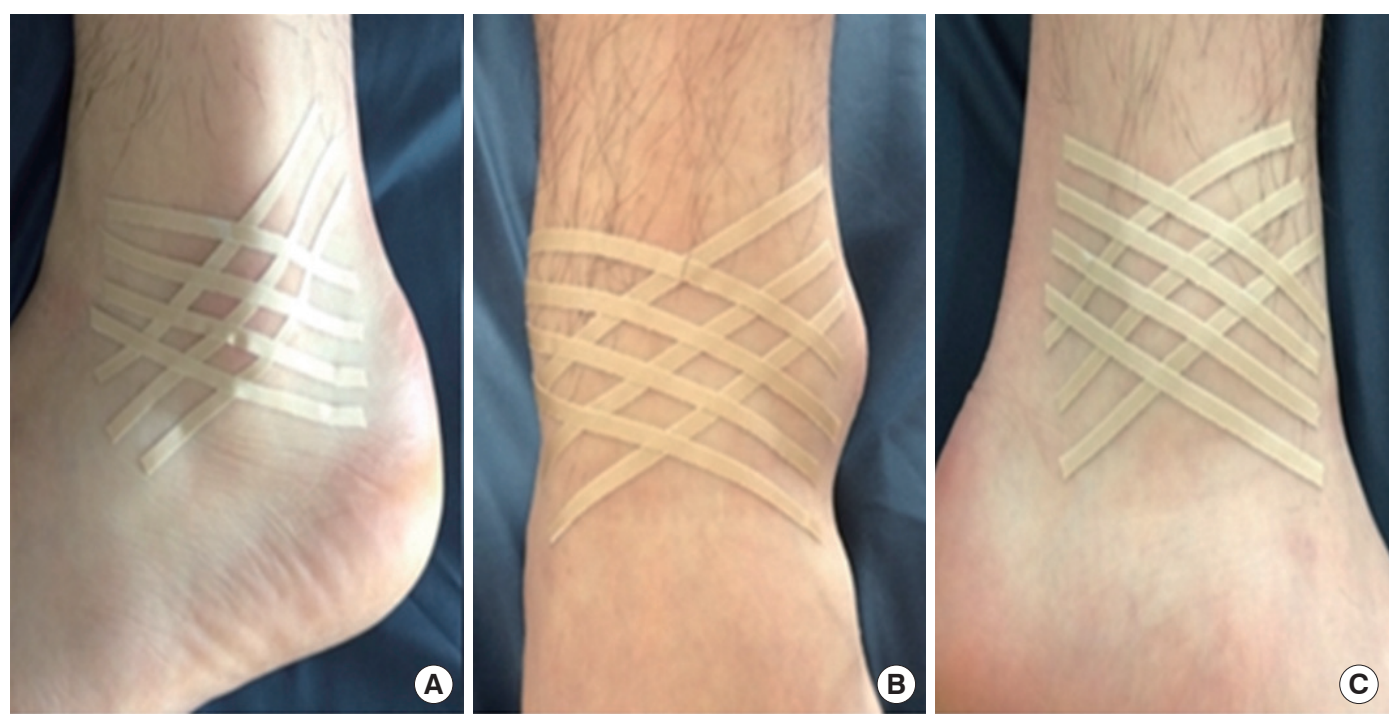

Figure 1. The method of Spiral taping application. (A) medial malleolus; (B) lateral malleolus; (C) anterior tibiotalar joint. 
the tape of $3 \times 4$ cross shape was attached on the lateral malleolus, similar to the medial malleolus (Figure 1B). (3) Finally, the tape of 3 $\times 4$ cross shape was attached on the anterior tibiotalar joint line. The tape was attached at the center of the joint line diagonally toward the right at a $30^{\circ}-35^{\circ}$ angle, and 3 tapes application were similar to that at the medial and lateral malleolus (Figure 1C). This taping protocol was selected because a majority of the population that would use tape therapy has left-oriented activity, whereas 1\%-5\% of the population has right-oriented activity. ${ }^{16}$ While attaching the tape to the ankle, the ankle was placed in a neutral position to avoid stretching the tape.

\section{Outcome Measurements}

Test for measuring dynamic balance is SEBT in musculoskeletal impairments such as chronic ankle instabilities. ${ }^{17-19}$ The intra-rater reliability of SEBT is very high $\left(\mathrm{ICC}_{2,1}=0.81-0.96\right) .{ }^{19}$ We performed SEBT as follows: (1) a 10-cm-wide tape was attached on the floor on the anteroposterior portion; (2) the tape was vertically attached on the mediolateral portion compared with the center of the anteriorposterior portion; (3) the tape was attached at a $45^{\circ}$ angle from the outside and inside of the anterior-posterior portion (all tape lengths were $180 \mathrm{~cm}$ ); and (4) while the subject was maintaining a barefoot single-leg stance in the center of 8 lines, the toe of the contralateral leg was placed far from the 8 lines and the distance between the center and the toe was measured. Because the trunk and pelvic are excessively rotated because of ankle instability during SEBT, ${ }^{21}$ the therapists were trained to prevent the rotation of the trunk and pelvis during SEBT. The better value from among two measurements was selected, and the unit measure is centimeter. SEBT value is sum of 8trials.

\section{Design and Procedure}

This study used a pre-post measures experimental design. The compared baseline and post-intervention changes, and compared difference stable ankle and unstable ankle in the subjects

Before conducting experimental test, the subjects were checked to determine whether they met the inclusion or exclusion criteria and the general characteristics of all subjects were recorded. Ankle instability was determined using the Cumberland ankle instability tool (CAIT) questionnaire, which is used to evaluate CAI ${ }^{22}$ CAIT questionnaire consists of 9 questions rated on a scale of 0-3 points, $0-4$ points, and 0-5 points, with the total score ranging from 0 to 30 points. Scores of $\geq 28$ indicate stability, whereas scores of $\leq 23$ indicate functional ankle instability. ${ }^{23}$ CAIT is a simple, valid, and reliable tool to measure the severity of ankle instability (validity: 0.83; reliability: 0.99), and the test-retest reliability is excellent [intra class correlation coefficient $\left.\left(\mathrm{ICC}_{2,1}\right)=0.96\right]^{22}$

CAIT questionnaire, SEBT were performed in Physical therapy laboratory and took approximately 2 hours. Subjects were training for the protocol of SEBT was completed. Thereafter, ST intervention was applied to the unstable ankle. Before and 30 minutes later after intervention measured SEBT on stable ankle and unstable ankle.

\section{Statistical Analysis}

All statistical analyses were performed using the statistical program SPSS 21.0 (Stastistical Package for the Social Sciences Inc, Chicago, IL) Analysis of descriptive statistics was used for the subject's general characteristics. Changes between pre- and post-intervention values, and comparison the difference of stable and unstable ankle were compared using paired t-test. In this study, quantitative measure of the strength of a pre- and post-intervention used Effect size (ES). All values are expressed as mean \pm standard deviation (SD). The significance level was set at $\mathrm{p}<0.05$.

\section{RESULTS}

Descriptive baseline characteristics, unstable ankle scores, duration, and CAIT scores are presented in Table 1. Table 2 shows that unstable ankle before and after ST application significantly improved $(\mathrm{p}<0.01, \mathrm{ES}=0.74)$. There were significantly differences between the unstable and stable ankles before and after intervention $(\mathrm{p}<0.01, \mathrm{ES}=1.88)$.

Table 1. General characteristics of the subjects

\begin{tabular}{lc}
\hline Gender (male/female) & $39(16 / 23)$ \\
\hline Age (year) & $21.44 \pm 1.66$ \\
Height $(\mathrm{cm})$ & $166.38 \pm 7.75$ \\
Weight $(\mathrm{kg})$ & $60.46 \pm 9.76$ \\
Affected leg (left/right) & $14 / 25$ \\
Duration (month) & $10.36 \pm 7.55$ \\
CAIT score & $19.56 \pm 3.29$ \\
\hline
\end{tabular}

CAIT: Cumberland ankle instability tool. 
Table 2. Star excursion balance test before and after the intervention

\begin{tabular}{lrrrrr}
\hline Variable (unit) & \multicolumn{1}{c}{ Pre-test } & Post-test & Change $(95 \% \mathrm{Cl})$ & $\mathrm{p}$ & Effect size \\
\hline Unstable ankle $(\mathrm{cm})$ & $51.54 \pm 9.85$ & $58.01 \pm 7.51$ & $6.35 \pm 2.57(5.47-7.24)$ & $0.000^{*}$ & 0.74 \\
Stable ankle $(\mathrm{cm})$ & $60.49 \pm 7.80$ & $60.67 \pm 8.74$ & $0.20 \pm 0.72(-0.45-0.45)$ & 0.109 & 0.02 \\
Difference & $9.08 \pm 4.00$ & $2.92 \pm 2.35$ & $6.16 \pm 2.88(5.16-7.14)$ & $0.000^{*}$ & 1.88 \\
\hline
\end{tabular}

${ }^{*} p<0.01$ compared with pretest and unstable ankle.

\section{DISCUSSION}

In this study investigated the effect of compared to pre- and post ST application on SEBT performance as dynamic balance in individuals with CAI.

Taping is very useful alternative method in CAI. Previous studies demonstrated that non-elastic tape suggest that ankle support is increased compared with elastic tape therapy. ${ }^{24,25}$ For dynamic balance measure, researchers and clinicians use SEBT reach distance to evaluate ankle instability, ${ }^{18}$ and SEBT reach distance is known to decrease in CAI. ${ }^{26}$ In this study we observed that SEBT of stable and unstable ankle was significant difference. The neuromuscular training program ${ }^{11}$ and balance training ${ }^{20}$ enhanced SEBT in CAI. ${ }^{11}$ SEBT improved that means to improved dynamic balance.

Current study we observed that performance of SEBT significantly increased after ST application. This finding suggested that ST enhanced ankle stability, and may reduce apprehension. The result of study was identical from former study showing mulligan taping, such as non-elastic taping, improves performance of SEBT. ${ }^{10}$ Therefore, the author suggested that application of ST was positive effect on performance of SEBT in CAI.

In the comparison of two means, ES of 0.20 is considered a small effect, 0.50 is considered a medium effect, and 0.80 is considered a large effect. ${ }^{26}$ In this study, large ES were observed on SEBT of unstable ankle and difference of unstable and stable ankle. ES is successful in detecting intervention effect and identifying the participants with the largest effect. ${ }^{27}$ Therefore, Improved SEBT performance of ankle may be contributes by ST application.

In this study, we showed that SEBT reach distance increase after ST application on unstable ankle. Therefore, because SEBT requires continuous movement of the foot other than the one supported to touch in eight directions, which also requires ankle stability, and dynamic balance control. ${ }^{28}$ Therefore, Increase in SEBT could suggest the occurrence of increase ankle stability.

As seen above, ST application in CAI increased SEBT as dynamic balance. Therefore, author suggests that ST may be a suitable intervention to improve ankle stabilization in individual with CAI in clinical setting.

Some limitation of this study is that the relatively low number of subjects makes it difficult generalized the result. And the long term effects of ST were not investigated, as the test were conducted immediately after applying the tape. Furthermore, the mechanism of the increase in proprioception and SEBT observed is also unclear. Also, previous study reported that ankle proprioception plays on essential role in balance control. ${ }^{29}$ This study suggests that further study should attempt to investigate effect of ST on several symptoms, such as increase pain, and decrease ROM and posture stability and muscle activity in individual with CAI.

\section{REFERENCES}

1. Hiller CE, Kilbreath SL, Refshauge KM. Chronic ankle instability: evolution of the model. J Athl Train. 2011;46(2):133-41.

2. van Rijn RM, van Os AG, Bernsen RM et al. What is the clinical course of acute ankle sprains? A systematic literature review. Am J Med. 2008; 121(4):324-31.e6

3. Gerber JP, Williams GN, Scoville CR et al. Persistent disability associated with ankle sprains: A prospective examination of an athletic population. Foot Ankle Int. 1998;19(10):653-60.

4. Delahunt E, Coughlan GF, Caulfield B et al. Inclusion criteria when investigating insufficiencies in chronic ankle instability. Med Sci Sports Exerc. 2010;42(11):2106-21.

5. Hertel J. Functional anatomy, pathomechanics, and pathophysiology of lateral ankle instability. J Athl Train. 2002;37(4):364-75.

6. Arnold BL, De La Motte S, Linens S et al. Ankle instability is associated with balance impairments: A meta-analysis. Med Sci Sports Exerc. 2009;41(5):1048-62.

7. Wikstrom EA, Tillman MD, Chmielewski TL et al. Dynamic postural stability deficits in subjects with self-reported ankle instability. Med Sci Sports Exerc. 2007;39(3):397-402.

8. Plisky PJ, Gorman PP, Butler RJ et al. The reliability of an instrumented device for measuring components of the star excursion balance test. North American journal of sports physical therapy: NAJSPT. 2009;4(2):92.

9. Kinzey SJ, Armstrong CW. The reliability of the star-excursion test in assessing dynamic balance. J Ortho\& Sports Phys Ther. 1998;27(5):35660. 
10. Olmsted LC, Carcia CR, Hertel J et al. Efficacy of the star excursion balance tests in detecting reach deficits in subjects with chronic ankle instability. J Athl Train. 2002;37(4):501.

11. Hong MS, Woo JH, Jung SH.The effect of neuromuscular training programme on dynamic balance of women basketball players with chronic ankle instability. J Korean Acad Ther. 2013;5:86-91.

12. Someeh M, Norasteh AA, Daneshmandi H et al. Immediate effects of mulligan's fibular repositioning taping on postural control in athletes with and without chronic ankle instability. Phys Ther Sport. 2015;16(2):135-9.

13. Lee BG, Lee JH. Immediate effects of ankle balance taping with kinesiology tape on the dynamic balance of young players with functional ankle instability. Technol Health Care. 2015;23(3):333-41.

14. Mickel TJ, Bottoni CR, Tsuji G et al. Prophylactic bracing versus taping for the prevention of ankle sprains in high school athletes: A prospective, randomized trial. J Foot \& Ankle Surg. 2006;45(6):360-5.

15. Danaka N. Spiral balance taping therapy. Gangju. Pyungwha ME CO.1997.

16. Lim C, Park Y, Bae Y. The effect of the kinesio taping and spiral taping on menstrual pain and premenstrual syndrome. J Phys Ther Sci. 2013; 25(7):761-4.

17. Hwang JO, Seo JC. The effects of spiral taping treatment on low back pain. Journal of Pharmacopuncture. 2006;9(1):103-7.

18. Lee BH, Lee HR, Kim KM et al. Effects of spiral taping applied to the neck and ankle on the body balance index. J Phys Ther Sci. 2015;27(1):79-82.

19. Hertel J, Miller SJ, Denegar CR. Intratester and intertester reliability during the star excursion balance tests. JSR. 2010;9(2):104-16.

20. Cha SH, Kim JS. The effect of balance on functional ankle stability with ankle sprained patients. Health \& Sports Med. 2009;11(2):73-83.
21. de la Motte S. Trunk-rotation differences at maximal reach of the star excursion balance test in participants with chronic ankle instability. J Athl Train. 2015;50(4):358-65.

22. Hiller CE, Refshauge KM, Bundy AC et al. The Cumberland ankle instability tool: a report of validity and reliability testing. Arch Phys Med Rehabil. 2006;87(9):1235-41.

23. de Noronha M, Refshauge KM, Kilbreath SL et al. Loss of proprioception or motor control is not related to functional ankle instability: an observational study. Aust J Physiother. 2007;53(3):193-8.

24. Briem K, Eythörsdöttir H, Magnúsdóttir RG et al. Effects of kinesio tape compared with nonelastic sports tape and the untaped ankle during a sudden inversion perturbation in male athletes. J Orthop Sports Phys Ther, 2011;41(5):328-35.

25. Kuni B, Mussler J, Kalkum E et al. Effect of kinesiotaping, non-elastic taping and bracing on segmental foot kinematics during drop landing in healthy subjects and subjects with chronic ankle instability. Physiother. 2016;102(3):287-93.

26. Olive ML, Smith BW. Effect size calculations and single subjects designs. Educ Psychol. 2005;25(2-3):313-24.

27. Cohen J. Statistical power analysis for the behavioral science. 2 nd ed, Lawrence Erlbaum Associates, New York, 1998:29.

28. Hertel J, Braham RA, Hale SA et al. Simplifying the star excursion balance test: analyses of subjects with and without chronic ankle instability. J Orthop Sports Phys Ther. 2006:36(3);131-7.

29. Han J, Anson J, Waddington G et al. The role of ankle proprioception for balance control in relation to sports performance and injury. Biomed Res Int. 2015;2015:842804. 